\title{
INFLUÊNCIA DA COMPOSIÇĀO QUIMIICA DE SOLUÇÕES SIMULADOS DOS POROS DE CONCRETO NA QUALIDADE DO FILME DE PASSIVAÇÃO DE AÇOS DE BAIXO TEOR DE CARBONO
}

\section{G. MODESTI}

Mestrando, Engenheiro Civil

Universidade do Vale do Rio dos Sinos

Rio Grande do Sul, Brasil

gmodesti@avalisinos.com.br

\author{
M. MANCIO \\ Prof. Dr., Engenheiro Civil \\ Universidade do Vale do Rio dos Sinos \\ Rio Grande do Sul, Brasil \\ mancio@unisinos.br
}

\author{
L. KREIN \\ Mestrando, Engenheiro Civil \\ Universidade do Vale do Rio dos Sinos \\ Rio Grande do Sul, Brasil \\ laerciokrein@gmail.com
}

\begin{abstract}
RESUMO
A corrosão é a principal manifestação patológica em estruturas de concreto armado. $\mathrm{O}$ estudo analisou a qualidade do filme de passivação de aços de baixo teor de carbono em soluções simulados de poros de concreto. Estudaram-se duas soluções, de pH 12,6 representativa de concretos com adição de 10\% de sílica ativa e de pH 13,6 de concreto com CPIIZ sem substituição, com alteração também da força iônica. Para controle da força iônica, utilizou-se $\mathrm{K}_{2} \mathrm{SO}_{4} \mathrm{e} \mathrm{C}_{6} \mathrm{H}_{5} \mathrm{~K}_{3} \mathrm{O}_{7}$. A análise se deu através dos ensaios de potencial de circuito aberto, resistência linear de polarização, voltametria cíclica e polarização catódica galvanostátiva. Os ensaios indicaram a formação de um melhor filme de passivação nas soluções de pH 12,6 com a utilização de citrato de potássio do que em pH 13,6 (sem adição), indicando a influência da força iônica em conjunto com o pH na qualidade do filme de passivação. A utilização de K2SO4 provocou a despassivação dos aços neste mesmo $\mathrm{pH}$, indicando a influência do sulfato na corrosão.
\end{abstract}

Palavras-chave:corrosão, filmes de passivação, força iônica, ensaios eletroquímicos, concreto armado.

\begin{abstract}
Reinforcement corrosion is the main pathological manifestation in reinforced concrete structures. This paper focuses on the analyses of the quality and behavior of passivation films of low carbon steel embedded in simulated concrete pore solutions. Two different solutions were studied, one with $\mathrm{pH} 12,6$ representative of concretes with $10 \%$ of silica fume

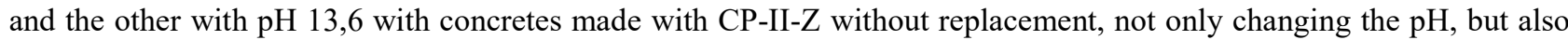
the ionic strength. To control the ionic strength, potassium sulfate and potassium citrate were used, with analysis of the different behavior between them. For that, electrochemical tests were used, such as open circuit potential, linear polarization resistance, cyclic voltammetry and cathodic galvanostatic polarization. The results indicated that a better passivation film was formed with the $\mathrm{pH} 12,6$ with potassium citrate than with a $\mathrm{pH}$ of 13,6, showing the influence of the ionic strength together with the $\mathrm{pH}$ in the quality of the passivation film. On the other hand, the use of potassium sulfate induced the corrosion of steel even with a $\mathrm{pH}$ of 12,6, indication the influence of sulfate ions in the corrosion.

Keywords: corrosion, passivation films, ionic strength, electrochemical tests, reinforced concrete.
\end{abstract}

\section{INTRODUÇÃO}

Toda matéria-prima do aço utilizado na construção civil é extraída da natureza sob forma de minério, estado mais estável termodinamicamente. Para obter-se um metal a partir do minério é necessária aplicação de energia térmica em grande quantidade no processo de metalurgia. De forma simples, pode-se explicar a corrosão como um processo de metalurgia na forma contraria, uma vez que a oxidação do metal gera um produto de corrosão com a composição idêntica ao minério encontrado na natureza. Além disso, o metal sempre tende a voltar à sua forma energética mais estável. (GENTIL, 1982; RAMANATHAN, 1988). 
Um dos principais fatores que podem desencadear um processo corrosivo em uma estrutura de concreto armado é a redução do $\mathrm{pH}$, causada geralmente pela carbonatação, devido a reações físico-químicas entre gases encontrados em ambientes urbanos, oriundos da queima de combustíveis fósseis e os componentes alcalinos do concreto. Os gases, principalmente o dióxido de carbono, reagem com o $\mathrm{Ca}(\mathrm{OH})_{2}, \mathrm{KOH}$ (hidróxido de potássio) e o $\mathrm{NaOH}$ (hidróxido de sódio), principais componentes alcalinos do concreto e responsáveis por manter o $\mathrm{pH}$ do concreto elevado e passivar a armadura. Com a penetração destes agentes agressivos, ocorre reação química, sendo esta a principal responsável por diminuir o pH do concreto em grandes centros urbanos. (HELENE, 1986; HUNKELER, 2005; BROOMFIELD, 2007; RIBEIRO et. al, 2014). Outro fator redutor do pH é a crescente utilização de adições minerais, pois, as adições pozolânicas reagem com os álcalis presentes na solução dos poros, consumindo a reserva alcalina dos concretos. (NEVILLE, BROOK, 2010; CASCUDO; CARASEK, 2011).

A fase líquida do concreto, denominada de soluções dos poros possui $\mathrm{pH}$ alcalino entre 12, e 13,5, tendo em vista seus principais constituintes. A solução é basicamente, composta por $\mathrm{NaOH}, \mathrm{KOH}$ e, em menor quantidade, $\mathrm{Ca}(\mathrm{OH})_{2}$ (POURSAEE, 2016; GHODS et al, 2009; BROOMFIELD, 2007, HAUSMANN, 2007). Apesar do Ca(OH) 2 também ser alcalino, o pH acima de 13 é mantido, majoritariamente, pelas hidroxilas provenientes do $\mathrm{NaOH}$ e KOH. (POURSAEE, 2010).

As características do cimento utilizado, presença de adições e substituições por materiais cimentícios suplementares, relação a/c ou a/agl, grau de hidratação e o meio externo influenciarão na composição da fase líquida dos poros. (KULAKOWSKI, 2002; SCOTT \& ALEXANDER, 2016). Neste sentido, diversos estudos são realizados através da extração da solução dos poros visando determinar a influência da solução na durabilidade de estruturas de concreto, como Plusquellec et al (2017), Scott \& Alexander (2016), Ortolan (2015), Kempl \& Çopuroglu (2015), Pu et al (2012), Kulakowksi (2002), Anderson et al (1989); Page \& Vennesland (1983). A utilização de soluções simuladas permite investigações mais precisas quanto à influência de composições químicas, pois possibilita um maior controle das variáveis em estudo (SCOTT; ALEXANDER, 2016).

A presença de íons na fase líquida do concreto, faz com que a solução se trate de um eletrólito, que possuirá uma determinada força iônica, dependendo da sua composição. A carga dos íons, se simples ou múltipla e a concentração dos mesmos determinará a força iônica (IS), pois com o aumento da concentração de íons, tem-se o aumento da força iônica. Esta característica da solução interfere na solubilidade de íons (SKOOG et al, 2006), podendo ser determinada através da Equação 1.

$$
I S=\frac{1}{2} \sum_{1}^{n} \mathrm{Zi}^{2} \mathrm{Ci}
$$

Onde:

IS = Força iônica;

$\mathrm{Zi}=$ Carga dos íons;

$\mathrm{Ci}=$ Concentração molar (mol/l)

Com o aumento da quantidade de íons diferentes em uma solução (e, consequentemente, da força iônica), ocorre o efeito de blindagem, onde íons com uma determinada carga, positiva ou negativa, são envoltos por íons de carga oposta (ATKINS; PAULA, 2012). Como consequência, tem-se um efeito que aparenta que íons positivos sejam menos positivos e os negativos menos negativos (SKOOG et al, 2006). Conforme Mancio et al (2009) e Ortolan (2015), quanto maior a força iônica de uma determinada solução, pior será a qualidade do filme de passivação. Considerando que a IS tende à aumentar com o aumento do $\mathrm{pH}$, dentro do espectro de $\mathrm{pH}$ que permite a passivação de armaduras, quanto maior o $\mathrm{pH}$, menor será a qualidade deste filme (MANCIO et al, 2009).

A composição da fase líquida do concreto tem influência na qualidade do filme de passivação, pois as ligas metálicas, em determinado ambiente, não corroem. Isso ocorre devido a formação películas protetoras finas e oxidadas. Essas camadas, denominadas de filme de passivação, são as principais responsáveis por dar as liga a resistência contra a corrosão. Basicamente, define-se passivação como a condição resistente à corrosão a partir de um filme de superfície de óxido hidratado fino, protetor, que atua como uma barreira à reação de dissolução anódica. (PAGE, 2007).

O filme de passivação é originado através que uma rápida reação eletroquímica, onde os produtos de corrosão gerados se agrupam na superfície do aço. A maioria dos pesquisadores adota o filme de passivação como uma estrutura perfeita composta por duas camadas, a interna formada por $\mathrm{Fe}_{3} \mathrm{O}_{4}$ e a camada externa formada por $\mathrm{Fe}(\mathrm{OH})_{2}$. Acredita-se que a camada externa é altamente defeituosa e condutiva. Além dos elementos citados acima, o filme de passivação pode conter 
elementos oriundos das ligas metálicas, que são responsáveis por melhorar suas propriedades. (JONES, 1992; GHODS et. al. 2012; GUNAY el. al. 2013). Conforme Mancio et al (2009), filmes com maior espessura possuem menor qualidade do que filmes menos espessos.

Visando facilitar o estudo de corrosão, vem-se utilizando soluções simuladas que imitam a composição e o pH das soluções dos poros, pois, o ambiente alcalino garantido pela solução dos poros é propicio à formação do filme de passivação. Um metal inserido em soluções dos poros simulados leva 7 dias para formar um filme de passivação estável, que iniba a corrosão (POURSAEE, HANSSON, 2007).

O presente trabalho tem como objetivos demonstrar a influência conjunta da força iônica e pH na qualidade do filme de passivação através de ensaios eletroquímicos.

\section{MATERIAIS E MÉTODOS}

\subsection{Materiais e amostras}

Foram utilizados vergalhões de aço de baixo teor de carbono do tipo CA-50S produzido pela Gerdau S.A. As barras utilizadas possuíam diâmetro $10 \mathrm{~mm}$, sendo elaboradas, cuja composição, conforme previsto na ABNT NBR 8965:1985 é indicada na Tabela 1. Para os ensaios as extremidades foram isoladas com resina epóxi, para fixar a área de estudo na lateral da barra. A área de ensaio era de $5,9 \mathrm{~cm}^{2}$, determinada através da digitalização tridimensional à lazer, realizada no Laboratório de Design e Seleção de Materiais da Universidade Federal do Rio Grande do Sul. Os vergalhões foram utilizados sem polimento da superície, tendo em vista que a alteração da superfície do aço através de usinagem (apesar de fornecer uma área de ensaio mais precisa) provoca alterações nos resultados de teor máximo de cloretos para despassivação (WHEAT et al., 1997; FIGUEIRA et al, 2017). Portanto, as amostras foram limpas com solvente, conforme previsto na ABNT NBR 15158:2016, e álcool isopropílico antes da imersão em solução.

Tabela 1: Composição do Aço CA-50

\begin{tabular}{c|c|c|c|c|c|c|c}
\hline Norma & Aço & $\% \mathrm{C}$ & $\% \mathrm{Mn}$ & $\% \mathrm{Si}$ & $\% \mathrm{P}$ & $\% \mathrm{~S}$ & $\% \mathrm{Cr}$ \\
\hline $\begin{array}{c}\text { ABNT NBR } \\
8965: 1985\end{array}$ & $\mathrm{CA}-50$ & 0,35 & 1,5 & 0,5 & 0,05 & 0,05 & - \\
\hline
\end{tabular}

Fonte: ABNT NBR 8965:1985

Durante os ensaios os aços foram imersos em soluções simuladas dos poros de concreto produzidas com água deionizada e adições dos reagentes P.A. $\mathrm{NaOH}, \mathrm{KOH}, \mathrm{K}_{2} \mathrm{SO}_{4}$ e $\mathrm{C}_{6} \mathrm{H}_{5} \mathrm{~K}_{3} \mathrm{O}_{7}$. Apresenta-se na Tabela 2 a composição das soluções propostas para este estudo. Utilizou-se como referência as soluções extraídas por Ortolan (2015), tendo em vista se tratarem de concretos produzidos na mesma região dos Autores deste artigo (região metropolitana de Porto Alegre/RS), mesmo havendo variabilidade do cimento. Os reagentes $\mathrm{K}_{2} \mathrm{SO}_{4}$ e $\mathrm{C}_{6} \mathrm{H}_{5} \mathrm{~K}_{3} \mathrm{O}_{7}$ foram utilizados, mesmo não fazendo parte de soluções reais, pois permitem adequar a força iônica das soluções simuladas àquelas reais sem alteração do pH. Este procedimento também foi realizado por Mancio et al (2009). Estudos com a utilização de íons $\mathrm{SO}_{4}{ }^{-2}$ também foram realizados por Xu et al (2020), Ogunsanya \& Hansson (2019) e Williamson \& Isgor (2016). A utilização de $\mathrm{C}_{6} \mathrm{H}_{5} \mathrm{~K}_{3} \mathrm{O}_{7}$ não tem por objetivo simular um inibidor de corrosão, apenas compensar a IS. Explica-se que as soluções extraídas não possuem quantidade de hidroxilas equivalente aos álcalis, impossibiltando a sua reprodução direta em laboratório, fazendo com que haja a necessidade de utilização destes reagentes para compensação e adequação à valores reais. Não foi utilizado $\mathrm{Ca}(\mathrm{OH})_{2}$, tendo em vista sua baixa solubilidade e teor de menor relevância frente aos demais hidróxidos extraídos.

Tabela 2: Composição, pH e IS das soluções reproduzidas

\begin{tabular}{|c|c|c|c|c|c|c|c|c|c|c|}
\hline \multirow{2}{*}{ Solução } & \multirow{2}{*}{$\begin{array}{c}\text { Adaptado } \\
\text { de: }\end{array}$} & \multirow{2}{*}{$\begin{array}{l}\mathrm{pH} \\
\text { Ref. }\end{array}$} & \multirow{2}{*}{$\begin{array}{c}\mathrm{pH} \\
\text { utilizado }\end{array}$} & \multicolumn{4}{|c|}{$\mathrm{Mol} / 1$} & \multirow{2}{*}{$\begin{array}{c}\text { IS } \\
\text { utilizado }\end{array}$} & \multirow{2}{*}{$\begin{array}{c}\text { IS } \\
\text { Ref. }\end{array}$} & \multirow{2}{*}{$\begin{array}{c}\text { Solub. } \\
\mathrm{Ca}(\mathrm{OH}) 2 \\
(\mathrm{M})\end{array}$} \\
\hline & & & & $\mathrm{NaOH}$ & $\mathrm{KOH}$ & $\mathrm{K} 2 \mathrm{SO} 4$ & C6H5K3O7 & & & \\
\hline 1 & $\begin{array}{c}\text { Ortolan } \\
(2015)\end{array}$ & 13,64 & 13,6 & 0,10 & 0,30 & 0 & 0 & 0,40 & 0,38 & 0,00004 \\
\hline 2 & $\begin{array}{l}\text { Ortolan } \\
(2015)\end{array}$ & 12,62 & 12,6 & 0,01 & 0,03 & 0,015 & 0 & 0,09 & 0,09 & 0,00267 \\
\hline 3 & $\begin{array}{l}\text { Ortolan } \\
(2015)\end{array}$ & 12,62 & 12,6 & 0,01 & 0,03 & 0 & 0,0084 & 0,09 & 0,09 & 0,00267 \\
\hline
\end{tabular}


A solução 1 é representativa de concreto produzido com cimento CP II-Z Já as soluções 2 e 3 são representativas de concreto produzido com cimento CP II-Z, havendo substituição deste por $10 \%$ de sílica ativa, oriunda da produção de silício metálico, do tipo não densificada. Ambos os concretos tiveram teor de argamassa de 55\%, com relação a/c 0,65. Os concretos foram produzidos utilizando agregado graúdo basáltico extraído em Campo Bom/RS e agregado miúdo do tipo areia natural de origem quartzosa extraída em Esteio/RS (ORTOLAN, 2015).

\subsection{Ensaios}

Utilizou-se a célula eletroquímica clássica de 3 eletrodos, cujo eletrodo de trabalho era a amostra de ação CA-50 descrita no item 2.1, complementado por contra-eletrodo em aço inoxidável e eletrodo de referência de $\mathrm{Ag} / \mathrm{AgCl}$. As células eletroquímicas utilizaram $500 \mathrm{ml}$ de solução simulada dos poros de concreto, atendendo a recomendação de quantidade mínima de $0,20 \mathrm{ml} / \mathrm{mm}^{2}$ de área de aço proposta pela NACE TM0169/G31 (NACE, 2012). Os eletrodos de trabalho foram imersos nas soluções por sete dias, de forma a garantir a formação de um filme de passivação estável (POURSAEE; HANSON, 2007, ZHIYONG et al, 2016).

Para análise da qualidade do filme de passivação foram realizados ensaios de Potencial de Circuito Aberto (também conhecido como potencial de corrosão), Resistência Linear de Polarização (RLP) e Voltametria Cíclica utilizando-se o potenciostato Autolab PGSTAT302N. Realizaram-se, também ensaios de Polarização Catódica Galvanostática, utilizando o potenciostato Gamry Intruments modelo Interface 1010E. Os ensaios de potencial de circuito aberto seguiram as recomendações da ASTM C876 (ASTM, 2015) e da RILEM (2003). Da ASTM (2015), utilizaram-se os valores absolutos de potencial, sendo potenciais ( $\mathrm{vs} \mathrm{Ag} / \mathrm{AgCl})>-106 \mathrm{mV}$ indicativos de baixo risco de corrosão $(<10 \%)$ ou passivação, potenciais $<-256 \mathrm{mV}$ indicativos de alto risco de corrosão $(>90 \%)$ ou despassivação. Valores intermediários são inconclusivos ou de risco médio. Já a análise pela RILEM, considera a queda de $-150 \mathrm{mV}$ em relação à amostras passivadas como representativas de despassivação.

Quanto ao RLP, aplicou-se uma tensão de +/- $30 \mathrm{mV}$ à uma velocidade de $0,1667 \mathrm{mV} / \mathrm{s}$, assim como proposto pela ASTM G59 (ASTM, 2014), verificando-se a alteração no potencial ( $\left.\mathrm{E}_{\text {corr }}\right)$ dos aços ensaiados. A resistência de polarização $\left(\mathrm{R}_{\mathrm{p}}\right)$ foi determinada através da Equação 2, utilizando o intervalo de $+/-10 \mathrm{mV}$. A análise dos resultados de resistência de polarização pode ser realizada, também, através de densidades de corrente, utilizando-se a Constante de Stern-Geary. A análise da taxa de corrosão é apresentada na Tabela 3. No presente estudo, considerou-se a Constante de Stern-Geary com valores de $6,2 \mathrm{mV}$ para o estado passivado e 14,7 $\mathrm{mV}$ para o estado despassivado, baseado em ensaios para determinação das curvas de Tafel realizados previamente.

Onde:

$$
\mathrm{R}_{\mathrm{p}}=\Delta \mathrm{E} / \Delta \mathrm{i}
$$

$\mathrm{R}_{\mathrm{p}}=$ Resistência de Polarização $(\Omega), \Delta \mathrm{E}=$ Variação de potencial (V) e $\Delta \mathrm{i}=$ Variação de corrente $(\mathrm{A})$

Tabela 3: Análise das densidades de corrente obtidas no ensaio de resistência de polarização.

\begin{tabular}{c|c|c}
\hline $\mathrm{I}_{\text {corr }}\left(\mu \mathrm{A} / \mathrm{cm}^{2}\right)$ & $\mathrm{V}_{\text {corr }}(\mathrm{mm} / \mathrm{ano})$ & Nível de corrosão \\
\hline$\leq 0,1$ & $\leq 0,001$ & Negligível \\
\hline $0,1-0,5$ & $0,001-0,005$ & Baixo \\
\hline $0,5-1,0$ & $0,005-0,010$ & Moderado \\
\hline$>1,0$ & $>0,010$ & Alto \\
\hline
\end{tabular}

Fonte: RILEM (2004, p.635). Traduzido pelos autores.

O ensaio de voltametria cíclica apresenta as características da formação e do rompimento do filme de passivação. A apresentação dos resultados dos ensaios é dívida em 3 zonas: (ABREU et al., 2004, CABRINI, LORENZI, PASTORES, 2014), quais sejam:

- Zona I: Na reação anódica, a densidade de corrente positiva permite a formação de íons, óxidos e hidróxidos: $\mathrm{Fe}^{+2}, \mathrm{Fe}_{3} \mathrm{O}_{4}, \mathrm{Fe}_{2} \mathrm{O}_{3}$. Na fase catódica, a densidade de corrente negativa provoca o consumo do filme de passivação formado na zona II.

- $\quad$ Zona II: Os óxidos, formados na zona I, originam o filme devido ao acréscimo do potencial;

- $\quad$ Zona III: O aumento da densidade de corrente, na fase anódica, forma a zona transpassiva, que é responsável por quebrar o filme. Porém, o filme é recomposto na reação catódica. 
A Voltametria Cíclica seguiu a metodologia descrita por Mancio et al (2009), no ensaio a varredura iniciando em um potencial de $-1,2 \mathrm{~V}$, onde o potencial foi aumentando até $0,7 \mathrm{~V}$ e posteriormente retorna até $-1,2 \mathrm{~V}$. A varredura do ensaio teve a sua taxa estipulada em $1 \mathrm{mV} / \mathrm{s}$. Mancio (2008) utilizou as equações 3 e 4 para calcular o potencial em que o $\mathrm{Fe}(\mathrm{OH})_{2}$ e o $\mathrm{Fe}_{3} \mathrm{O}_{4}$. Assume-se que o valor de $\mathrm{HFeO}_{2}$ é $1 \times 10^{-6}$

$$
\begin{aligned}
& \Delta_{\varnothing 2 e\left(\mathrm{Fe} \rightarrow \mathrm{Fe}(\mathrm{OH})_{2}\right.}=-0,047-0,0591 p \mathrm{H} \\
& \Delta_{\varnothing 2 e\left(\mathrm{HeO}_{2} \rightarrow \mathrm{Fe}_{3} \mathrm{OH}_{4}\right)}=-1,819+0,0295 \mathrm{pH}-0,0886 \log \left(\mathrm{HFeO}_{2}\right.
\end{aligned}
$$

Em relação à Polarização Catódica Galvanostáica, a corrente catódica foi aplicada e mantida constante em $-0,6 \mathrm{~mA} / \mathrm{cm}^{2}$ para reduzir o filme de passivação formado. A queda de potencial foi monitorada ao longo do tempo. A multiplicação de tempo (segundos) e potencial aplicado (V), dividido pela área superficial da amostra rende a carga elétrica $\left(\mathrm{C} / \mathrm{cm}^{2}\right)$ correspondente. $\mathrm{Da}$ análise do relação entre a queda no potencial e a carga elétrica aplicada, determinou-se a espessura do filme de passivação formado, através da Equação 5, considerando-se a camada interna do filme como uniforme e formada por $\mathrm{Fe}(\mathrm{OH})_{2}$ e a camada externa uniforme e formada por $\mathrm{Fe}_{2} \mathrm{O}_{3}$ (MANCIO et al, 2009).

$$
y=\frac{C . M W}{\delta . n . F}
$$

Onde:

$\mathrm{y}=$ espessura estimada do filme de passivação $(\mathrm{cm})$;

$\mathrm{C}=$ carga $\left(\mathrm{C} / \mathrm{cm}^{2}\right)$, resultado obtido pelo $\mathrm{PCG}$;

$\mathrm{MW}=$ massa molar da camada consumida $(\mathrm{g} / \mathrm{mol})$;

$\delta=$ densidade específica do oxido da camada $\left(\mathrm{g} / \mathrm{m}^{3}\right)$;

$\mathrm{F}=$ Constante de Faraday (96500 Coul/equivalente);

$\mathrm{n}=$ número de e-trocados (equivalentes $/ \mathrm{mol}$ ).

\section{RESULTADOS E DISCUSSÃO}

\subsection{Potencial de circuito aberto e Resistência de polarização}

Os resultados obtidos para o ensaio de potencial de circuito aberto são apresentados na Tabela 4. Constata-se que o potencial menos eletronegativo foi o da solução 2 , no valor de $-87 \mathrm{mV}$, seguido pela solução $1 \mathrm{com}-122 \mathrm{mV}$ e, por fim, a solução 3 com $-350 \mathrm{mV}$.

Tabela 4: Resultados obtidos no ensaio de potencial de circuito aberto

\begin{tabular}{c|l|c|l}
\hline Solução & \multicolumn{1}{|c|}{ Identificação } & $\begin{array}{c}\text { Ecorr } \\
(\mathrm{mV} \text { vs Ag/AgCl })\end{array}$ & \multicolumn{1}{c}{ Análise (ASTM C876) } \\
\hline 1 & $\mathrm{pH} 13,6$ & -122 & Zona inconclusiva \\
\hline 2 & $\mathrm{pH} 12,6-\mathrm{C}_{6} \mathrm{H}_{5} \mathrm{~K}_{3} \mathrm{O}_{7}$ & -87 & Passivado - probabilidade de corrosão $<10 \%$ \\
\hline 3 & $\mathrm{pH} 12,6-\mathrm{K}_{2} \mathrm{SO}_{4}$ & -350 & Despassivado - probabilidade de corrosão $>90 \%$ \\
\hline
\end{tabular}

Da análise pelos critérios da ASTM C876, nota-se que na solução 2 obteve-se resultados indicativos de passivação, enquanto a solução 3 apresentou potencial indicativo de $90 \%$ de probabilidade de corrosão, destoando dos demais. A variação entre os potenciais destas soluções é de $263 \mathrm{mV}$, superior à queda de $150 \mathrm{mV}$ indicados pela RILEM (2003) para comparativo entre amostras passivadas e despassivadas. Em relação à solução 1, os resultados estão próximos àqueles representativos de passivação, porém já na zona inconclusiva.

A Figura 1 demonstra a variação da tensão em relação à variação da corrente obtidos no ensaio de resistência linear de polarização. Nota-se que as retas obtidas para o pH 13,6 e 12,6 com utilização de $\mathrm{C}_{6} \mathrm{H}_{5} \mathrm{~K}_{3} \mathrm{O}_{7}$ apresentam-se praticamente verticais, enquanto a reta obtida para o $\mathrm{pH}$ 12,6 com $\mathrm{K}_{2} \mathrm{SO}_{4}$ apresenta-se próxima à horizontal. Considerando que um menor gradiente de corrente (reta vertical) se traduz numa maior Resistência de Polarização, tem-se que quanto mais verticalizada a reta, melhor será a qualidade do filme formado (Vide Equação 2).

A maior verticalidade da reta indica que o pH 12,6 com utilização de $\mathrm{C}_{6} \mathrm{H}_{5} \mathrm{~K}_{3} \mathrm{O}_{7}$ apresenta maior qualidade do filme de passivação do que o pH 13,6, devido à menor variação de corrente, resultando em uma maior resistência de polarização. 
Já quanto ao pH 12,6 com utilização de $\mathrm{K}_{2} \mathrm{SO}_{4}$ a horizontalidade destoante das demais indica que a mesma está em processo corrosivo. A variação da corrente entre +/- $10 \mathrm{mV}$ é de $0,55 \mu \mathrm{A}$ para a Solução 1 (pH 13,6), -0,37 $\mu \mathrm{A}$ para a Solução 2 (pH 12,6) e 18,42 para a Solução 3 (pH 12,6), confirmando a tendência de despassivação.

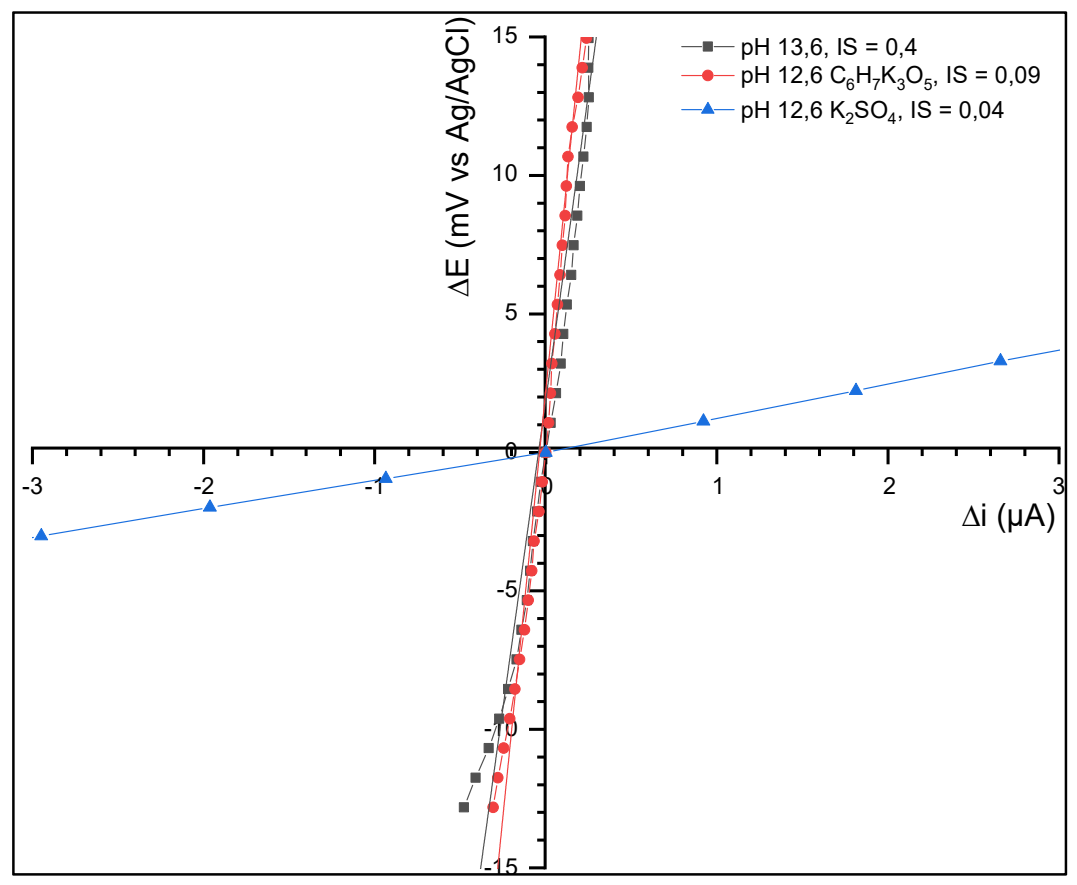

Figura 1: Resistência linear de polarização das soluções estudadas

A análise dos valores absolutos de $\mathrm{Rp}$ e densidade de corrente constam na Tabela 5. Para a solução 1 realizaram-se simulações com a Constante de Stern-Geary (B) com valor indicativo tanto de estado passivado quanto despassivado, tendo em vista a inconclusividade do ensaio de potencial de circuito aberto. Verifica-se que para ambos os cenários, as densidades de corrente são inferiores à $0,1 \mu \mathrm{A} / \mathrm{cm}^{2}$, determinando que a armadura está em estado passivo. Entretanto, as densidades são superiores àquela obtida para a solução 2 , confirmando a maior qualidade do filme de passivação formado em pH menor. Já em relação à solução 3, as densidades de corrente confirmam o estado de corrosão da armadura.

Tabela 5: Resultados obtidos no ensaio de Resistência Linear de Polarização.

\begin{tabular}{c|c|c|c|c|c|l}
\hline \multirow{2}{*}{ Solução } & Identificação & $\begin{array}{c}\mathrm{Rp} \\
(\Omega)\end{array}$ & $\begin{array}{c}\mathrm{Rp} \\
\left(\mathrm{k} \Omega \cdot \mathrm{cm}^{2}\right)\end{array}$ & $\begin{array}{c}\mathrm{B} \\
(\mathrm{mV})\end{array}$ & $\begin{array}{c}\text { Icorr } \\
\left(\mu \mathrm{A} / \mathrm{cm}^{2}\right)\end{array}$ & Análise \\
\hline \multirow{2}{*}{1} & $\mathrm{pH} \mathrm{13,6}$ & 39.273 & 232 & 6,2 & 0,026 & Passivado \\
\cline { 5 - 7 } & $\mathrm{pH} 12,6-\mathrm{C}_{6} \mathrm{H}_{5} \mathrm{~K}_{3} \mathrm{O}_{7}$ & 56.895 & 336 & 6,2 & 0,018 & Passivado \\
\hline 3 & $\mathrm{pH} 12,6-\mathrm{K}_{2} \mathrm{SO}_{4}$ & 1.140 & 6,7 & 14,7 & 2,178 & Despassivado \\
\hline
\end{tabular}

A análise conjunta dos ensaios de potencial de circuito aberto e resistência linear de polarização demonstram a formação de um filme de passivação de maior qualidade no $\mathrm{pH} 12,6$ com utilização de $\mathrm{C}_{6} \mathrm{H}_{5} \mathrm{~K}_{3} \mathrm{O}_{7}$ e IS = 0,09 do que em pH $13,6 \mathrm{e}$ IS =0,4. Resultados similares foram obtidos por Mancio et al (2009) e Ortolan (2015) com a diminuição do pH (dentro de valores que permitam a passivação) e da força iônica favorecendo a formação de filmes de passivação superiores.

Em relação à solução 3, com utilização de $\mathrm{K}_{2} \mathrm{SO}_{4}$ apesar de Mancio et al (2009) terem conseguido manter armaduras passivadas corrigindo o pH com este reagente, outros estudos como Liu et al (2016) e Xu et al (2019), indicam que a presença de sulfatos na solução prejudica a formação do filme de passivação, podendo levar à corrosão e, explicando os resultados destoantes para esta amostra. 


\subsection{Voltametria cíclica}

A Figura 2 apresenta gráfico da polarização cíclica do aço CA-50 com a solução 1. Nesse ensaio a amostra foi submetida a um potencial elétrico iniciado em $-1,2 \mathrm{~V}$ até $0,7 \mathrm{~V}$ e posteriormente retornando de $0,7 \mathrm{~V}$ até $1,2 \mathrm{~V}$. No início do ensaio, aplicar o potencial percebe-se a formação de um pico bem formado de oxidação próximo aos $-0,76 \mathrm{~V}$ e outros dos picos bem pequenos no potencial $-0,4 \mathrm{~V}$ e -0,145V. Já o filme e passivação é formado entre os potenciais de $-0,084 \mathrm{~V}$ até $0,52 \mathrm{~V}$. acima de 0,52 V percebe-se a formação de uma zona transpassiva. No período de retorno da varredura percebe-se dois picos que caracterizam a formação de duas camadas do filme de passivação nos potenciais $-0,956 \mathrm{~V}$ e $-1,2 \mathrm{~V}$, esse fenómeno havia sido observado por Mancio et al (2009).

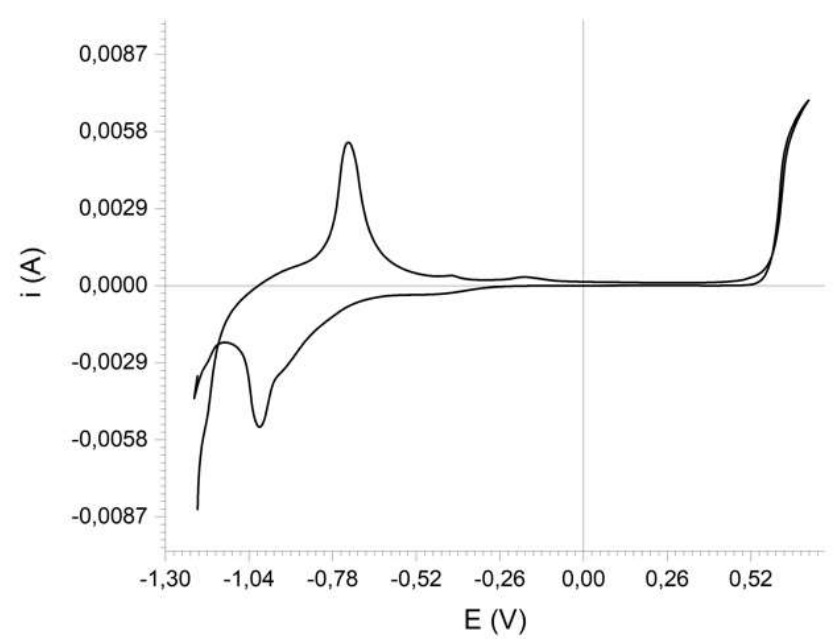

Figura 2: Voltametria Cíclica da solução 1

Já na Figura 3 (2) onde as amostras foram ensaiadas com solução 2, nota-se apenas um pico de oxidação no potencial de $-0,66 \mathrm{~V}$ e a formação do filme de passivação se da entre os potenciais de $-0,44 \mathrm{~V}$ até $0,52 \mathrm{~V}$, após se inicia a zona transpassiva. Já os picos formados no período de retorno ocorrem no potencial $-0,956 \mathrm{~V}$ e $-1,2 \mathrm{~V}$, mesmos potenciais do aço submetido ao pH 13,6. Nas amostras ensaiadas com solução 3, visto na Figura 3 (3), percebe-se certa similaridade com a Figura 3 (2), pois o pico de oxidação acontece em um potencial similar, embora a corrente seja menor. Outra similaridade ocorre na formação do filme de passivação e nos picos que indicam as camadas do filme de passivação.
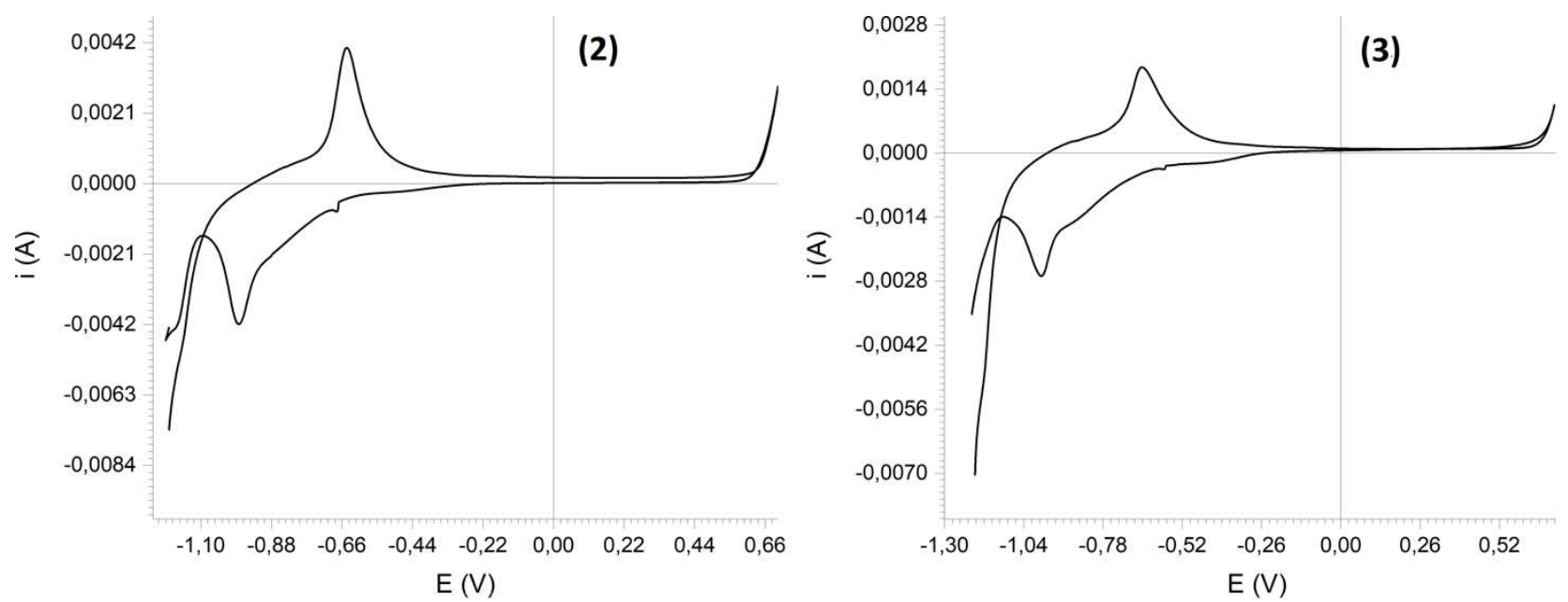

Figura 3: Voltametria Cíclica das soluções 2 e 3

As equações 3 e 4 consideram o valor do pH para estimar o potencial de formação do $\mathrm{Fe}(\mathrm{OH})_{2}$ e do $\mathrm{Fe}_{3} \mathrm{O}_{4}$. A solução 1 forma o $\mathrm{Fe}(\mathrm{OH})_{2}$ no potencial de $-0,85 \mathrm{Ve}$ o $\mathrm{Fe}_{3} \mathrm{O}_{4}$ no potencial de $-0,88 \mathrm{~V}$. As soluções 2 e 3 , de mesmo $\mathrm{pH}$, formam $\mathrm{Fe}(\mathrm{OH})_{2}$ e $\mathrm{Fe}_{3} \mathrm{O}_{4}$ nos potenciais de $-0,79 \mathrm{~V}$ e $-0,91 \mathrm{~V}$, respetivamente. 


\subsection{Polarização catódica galvanostática}

A Figura 4 apresenta a curva de polarização catódica galvanostática do aço CA-50 para o pH 13,6, nomeada como solução 1. A análise da curva de $\mathrm{pH} 13,6$ mostra duas inflexões nas curvas, a primeira camada começa a ser consumida na carga de $-0,2159 \mathrm{C} / \mathrm{cm}^{2}$ até $-0,14407 \mathrm{C} / \mathrm{cm}^{2}$. Com a equação 5 é possível estimar a espessura da camada externa em $168 \mathrm{~nm}$, já a camada interna tem espessura estimada de $301 \mathrm{~nm}$. Portanto, ao somar a espessura estimada das duas camadas, tem-se que a camada do filme de passivação é $469 \mathrm{~nm}$.

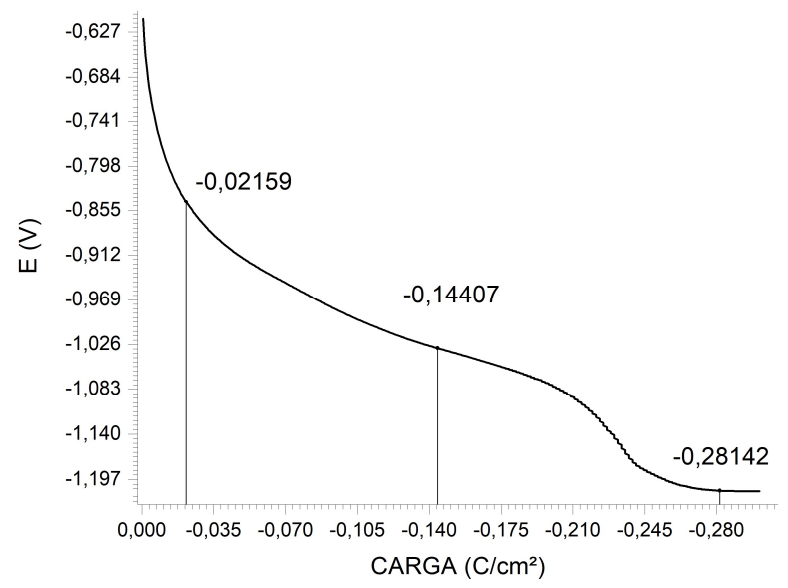

Figura 4: Polarização Catódica Galvanostática da solução 1

A Figura 5 apresenta as curvas de polarização catódica galvanostática do CA-50 para as soluções 2 e 3, com pH 12,6 respectivamente. A redução do $\mathrm{pH}$ solução 2, proporciona a redução da espessura estimada, a camada externa é consumida na faixa de carga entre $-0,0198 \mathrm{C} / \mathrm{cm}^{2}$ e $-0,1248 \mathrm{C} / \mathrm{cm}^{2}$ cuja espessura estimada da camada é de $144 \mathrm{~nm}$. Já a camada interna é consumida na faixa entre $-0,1248 \mathrm{C} / \mathrm{cm}^{2}$ e $-0,2112 \mathrm{C} / \mathrm{cm}^{2} \mathrm{com}$ espessura estimada de $136 \mathrm{~nm}$. A soma das espessuras estimadas das duas camadas, tem-se um filme de $280 \mathrm{~nm}$ de espessura.

A solução 3 altera a espessura estimada do filme de passivação, a faixa de carga de consumo da primeira camada varia entre $-0,01447 \mathrm{C} / \mathrm{cm}^{2}$ e $-0,10831 \mathrm{C} / \mathrm{cm}^{2}$, proporcionando espessura estimada de $129 \mathrm{~nm}$. Já a camada interna varia entre $-0,10831 \mathrm{C} / \mathrm{cm}^{2}$ e $-0,21612 \mathrm{C} / \mathrm{cm}^{2}$, gerando uma camada com espessura de $170 \mathrm{~nm}$. A soma das espessuras estimadas das duas camadas, tem-se um filme de $299 \mathrm{~nm}$ de espessura.

A redução da espessura do filme de passivação atrelado a redução do pH já foi observada por Mancio et al (2009), onde o autor notou uma melhora na qualidade protetora do filme de passivação. Por outro lado, a utilização do $\mathrm{K}_{2} \mathrm{SO}_{4}$, para aumentar a força iônica da solução não teve o resultada esperado, cujo reagente interfere diretamente na qualidade do resultado e ensaios devido a presença de ruídos na leitura do consumo do filme de passivação, como visto na Figura 5(3).
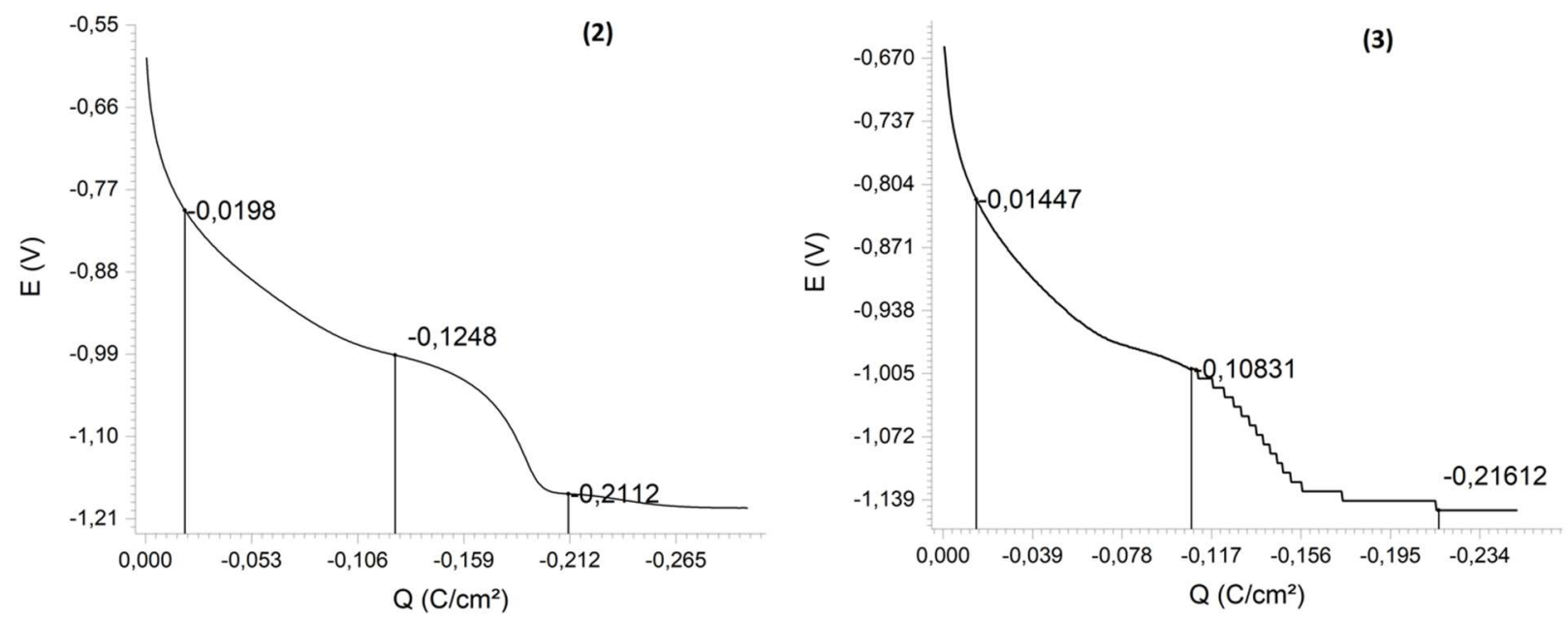

Figura 5: Polarização Catódica Galvanostática das soluções 2 e 3 


\section{CONCLUSÃO}

Os ensaios eletroquímicos realizados demonstraram a formação de um filme de passivação de melhor qualidade quando

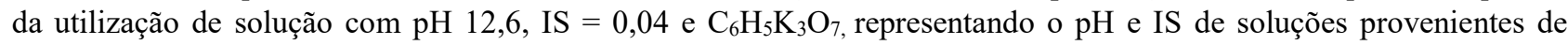
concretos com adição de materiais cimentícios suplementares, em relação à solução de $\mathrm{pH} 13,6$ e IS =0,9, representativa de soluções de concreto sem adições de materiais cimentícios suplementares. $\mathrm{O}$ ensaio de potencial de circuito aberto indicou uma menor probabilidade de corrosão, sendo esta informação confirmada através da resistência de polarização e densidade de corrente, comprovando a formação de um filme superior. A resistência de polarização na solução 2 foi $44 \%$ superior do que aquela da solução 1, resultando em uma densidade de corrente $31 \%$ inferior (ambas características de passivação).

Quanto aos ensaios de voltametria cíclica, verificou-se que ocorreram mudanças, principalmente, nos potenciais para formação das camadas de $\mathrm{Fe}(\mathrm{OH})_{2} \mathrm{e} \mathrm{Fe}_{3} \mathrm{O}_{4}$. Com a redução do $\mathrm{pH}$, a camada de $\mathrm{Fe}(\mathrm{OH})_{2}$ se formou em potenciais menos negativos e, portanto, foi originado mais tardiamente no ensaio. Diferentemente, a camada de $\mathrm{Fe}_{3} \mathrm{O}_{4}$ se formou em potencias mais negativos e se formou mais próximo ao inicio do ensaio. Os picos da fase anódica foram formados mais tarde e apresentaram menor amplitude quando da diminuição do $\mathrm{pH}$.

Já o ensaio de polarização catódica galvanostática confirmou a formação de filmes de menor espessura na solução 2 em relação à solução 1, colaborando para o entendimento de que a menor espessura resulta em uma maior qualidade do filme. O filme formado na amostra exposta à solução 2 apresentou espessura $40 \%$ inferior àquela formada na amostra da solução 1. Já em relacionando as soluções 2 e 3 percebe-se um pequeno aumento da espessura, o filme de passivação formado na solução 3 é cerca de 7\% mais espesso que o filme de passivação da solução 2 .

A análise conjunta da força iônica e pH para determinação das características das soluções dos poros de concreto tornase importante para a correta reprodução de soluções extraídas dos poros de concreto, evitando discrepâncias entre soluções reais e simuladas. Ainda, os resultados obtidos, colaboram para o entendimento de que a utilização de materiais cimentícios suplementares, apesar de provocarem a queda do $\mathrm{pH}$, não prejudicam a passivação das armaduras. Pelo contrário, favorecem a formação de filmes de melhor qualidade pela diminuição da força iônica que acompanha a diminuição do $\mathrm{pH}$.

Em relação à amostra exposta à solução 3 , a mesma restou prejudicada e despassivada com a utilização de $0,015 \mathrm{Mol} / \mathrm{L}$ de $\mathrm{K}_{2} \mathrm{SO}_{4}$. A despassivação está provavelmente relacionada à presença de sulfatos que alteraram as características do filme de passivação. assim como já identificado por Xu et al (2020) e Liu et al (2016).

\section{AGRADECIMENTOS}

Os autores agradecem a colaboração dos funcionários e disponibilização de equipamentos do Laboratório de Química e Farmácia e Laboratório de Caracterízação e Valorização de Materiais, bem como o Instituto Tecnológico ITT Chip, todos da Universidade do Vale do Rio dos Sinos - UNISINOS.

\section{REFERÊNCIAS}

ABREU, C. M., CRISTÓBAL, M. J., LOSADA, R., NÓVOA, X. R., PENA, G., \& PÉREZ, M. C. Comparative study of passive films of different stainless steels developed on alkaline medium. Electrochimica Acta, v. 49, n. 17-18, p. 30493056, 2004.

ANDERSSON, K.; ALLARD, B.; BENGTSSON, M., MAGNUSSON, C. Chemical composition of cement pore solutions. Cement and Concrete Research, v. 19. p. 327-332, 1989.

ASSOCIAÇÃO BRASILEIRA DE NORMAS TÉCNICAS. NBR 8965: Barras de aço CA 42 S com características de soldabilidade destinadas a armaduras de concreto armado. Rio de Janeiro, 1985.

ASSOCIAÇÃO BRASILEIRA DE NORMAS TÉCNICAS. NBR 15158. Limpeza de superfície de aço por compostos químicos. Rio de Janeiro, 2016.

ASTM INTERNATIONAL. ASTM C876: Standard Test Method for Corrosion Potentials of Uncoated Reinforcing Steel in Concrete. EUA, 2015. 
ASTM INTERnATIONAL. ASTM G59. Conducting Potentiodynamic Polarization Resistance Measurements. EUA, 2014.

ATKINS, P.; PAULA, J. Fisico-Química, 9 ed., Rio de Janeiro:LTC, 2012.

BROOMFIELD, J. P. Corrosion of steel in concrete: Understanding, investigation and repair. 2 ed., Londres, Inglaterra: E \& FN Spon, 2007.

CABRINI, M.; LORENZI, S.; PASTORE, T. Cyclic voltammetry evaluation of inhibitors for localized corrosion in alkaline solutions. Electrochimica Acta, v. 124, p. 156-164, 2014.

CASCUDO, O.; CARASEK, H. Ação da carbonatação no concreto. Concreto: Ciência e Tecnologia. São Paulo: IBRACON, 2011, v. 1p. 849-887.

SKOOG, D.A.; WEST, D.M.; HOLLER, F.J. Fundamentals of Analytical Chemistry, 8ed., Thomson Learning Ltda, 2006.

FIGUEIRA, R. B.; SADOVKSI, A.; MELO, A. P.; PEREIRA, E.V. Chloride threshold value to initiate reinforcement corrosion in simulated concrete pore solutions: The influence of surface finishing and $\mathrm{pH}$. Construction and Building Materials, v. 141, p. 183-200, 2017.

GENTIL, V. Corrosão: Livros Técnicos e Científicos Editora SA. Rio de Janeiro, 1996.

GHODS, P.; ISGOR, O.B.; BENSEBAA, F.; KINGSTON, D. Angle-resolved XPS study of carbon steel passivity and chloride-induced depassivation in simulated concrete pore solution. Corrosion Science, v. 58, p. 159-167, 2012.

GHODS, P.; ISGOR, O.B.; MCRAE, G.; MILLER, T. The effect of concrete pore solution composition on the quality of passive oxide films on black steel reinforcement. Cement \& Concrete Composites, v. 31, p. 2-11, 2009.

GHUNNAY, H.B.; GHODS, P.; ISGOR, O.B.; CARPENTER, G.J.C.; WU, X. Characterization of atomic structure of oxide films on carbon steel in simulated concrete pore solutions using EELS. Applied Surface Science, v. 274 , p. 195$202,2013$.

HAUSMANN, D.A. Three myths about corrosion of steel in concrete. Materials Performance, v. 46, p. 70-73, 2007.

HELENE, P. Corrosão em armaduras de concreto armado. São Paulo: Pini, 1986.

HUNKELER, F. Corrosion of reinforced concrete: processes and mechanisms. In: BÖHNI, H (ed.). Corrosion in reinforced concrete structures. EUA: CRC Press, 2005, p. 1-45.

KEMPL, J.; ÇOPUROGLU, O. The interaction of $\mathrm{pH}$, pore solution composition and solid phase composition of carbonated blast furnace slag cement paste activated with aqueous sodium monofluorophosphate. 15th Euroseminar on Microscopy Applied to Building Materials, Holanda, 2015.

KULAKOWSKI, M.P. Contribuição ao estudo da carbonatação em concretos e argamassas compostos com adição de sílica ativa, 2002. 199 f. Tese (Doutorado em Engenharia), Programa de Pós-Graduação em Engenharia de Minas, Metalúrgica e de Materiais, Universidade Federal do Rio Grande do Sul, 2002.

LIU, G.; ZHANG, Y.; NI, Z.; HUANG, R. Corrosion behavior of steel submitted to chloride and sulphate ions in simulated concrete pore solution. Construction and Building Materials, v. 115, p.1-5, 2016.

MANCIO, M. Electrochemical and in-situ Surface-Enhanced Raman Spectroscopy (SERS) study of passive films formed on low-carbon steel in highly alcaline environments. 2008. 221 f. Tese (Ph.D em Engenharia Civil e Engenharia Ambiental), University of California,Berkeley, 2008. 
MANCIO, M.; KUSINSKI, G.; DEVINE, T.M.; MONTEIRO, P.J.M. Electrochemical and in-situ SERS study of passive film characteristics and corrosion performance of microcomposite steel in simulated concrete pore solutions. University of California, Berkeley, 2009.

NATIONAL ASSOCIATION OF CORROSION ENGINEERS (NACE - International). TM 0169/G31: Standard Guide for Laboratory Immersion Corrosion Testing of Metals. Texas: NACE International, 2012.

NEVILle, A. M.; BROOKS, J. J. Tecnologia do Concreto. 2o. ed. Porto Alegre: Ruy Alberto Cremonini, 2013.

OGUNSANYA, I.G.; HANSSON, C.M. Influence of chloride and sulphate anions on the electronic and electrochemical properties of passive films formed on steel reinforcing bars. Materialia, v. 8, 2019.

ORTOLAN, V. Avaliação da influência do pH e da força iônica da solução dos poros do concreto na resistência à corrosão da armadura, 2015. 132 f. Dissertação (Mestrado em Engenharia Civil), Programa de Pós-Graduação em Engenharia Civil, Universidade do Vale do Rio dos Sinos, 2015.

PAGE, C.L.; VENESLAND, Ø. Pore solution composition and chloride binding capacity of sílica-fume cement pastes. Materials and Structures, v. 19, p.19-25, 1983.

PAGE, C.L.; VENESLAND, Ø. Pore solution composition and chloride binding capacity of sílica-fume cement pastes. Materials and Structures, v. 19, p.19-25, 1983.

PLUSQUELLEC, G.; GEIKER, M.R; LINDGARD, J.; DUCHESNE, J.; FOURNIER, B.; WEERDT, K. Determination of the $\mathrm{pH}$ and the free álcali metal contente in the pore solution of concrete: Review and experimental comparison.

Cement and Concrete Research, v. 96, p. 13-26, 2017.

POURSAEE, A. Corrosion of steel in concrete structures. In: POURSAEE, A. (ed). Corrosion of steel in concrete structures, Cambridge: Woodhead Publishing, p. 19-3, 2016.

POURSAEE, A. Corrosion of steel rebars in saturated $\mathrm{Ca}(\mathrm{OH}) 2$ and concrete pore solution. Concrete Research Letters, v. 1, p. $90-97$, set. 2010 .

POURSAEE, A.; HANSSON, C.M. R Reinforcing steel passivation in mortar and pore solution. Cement and Concrete Research, v. 37, p. 1127-1133, 2007.

PU, Q; JIANG, L; XU, J; CHU, H; XU, Y; ZHANG, Y. Evolution of pH and chemical composition of pore solution in carbonated concrete. Construction and Building Materials, v. 28, n.1, p. 519-524, mar. 2012.

RAMANATHAN, L. V. Corrosão e seu Controle. Hermus, São Paulo, 1986.

RIBEIRO, D. V. et. al. Corrosão em estruturas de concreto armado: Teoria, controle e métodos de análise. Rio de Janeiro: Campus, 2014.

RILEM TC 154-EMC. Recommendations: Half-cell potential measurements - Potential mapping on reinforced concrete structures. Materials and Structures, v. 36, p. 461-471, 2003.

SCOTT, A.; ALEXANDER, M.G. Effect of supplementary cementitious materials (binder type) on the pore solution chemistry and the corrosion of steel in alkaline environments. Cement and Concrete Research, v.89, p. 45-55, 2016.

WHEAT, H.G.; KASHTURIRANGAN, J.; KITOWSKI, C.J. Behaviour of steel in simulated concrete solutions. In: SCRIVENER, K.L.; YOUNG, J.F. Mechanisms of chemical degradation of cement-based systems. P. 143-150, 1997.

WILLIAMSON, J.; ISGOR, O. B. The effect of simulated concrete pore solution composition and chlorides on the electronic properties of passive films on carbon steel rebar. Corrosion Science, v. 106, p. 82-95, 2016.

XU, J.; TAN, Q.; MEI, Y. Corrosion protection of steel by Mg-Al layered double hydroxides in simulated concrete pore solution: Effect of SO42-. Corrosion Science, v. 163, 2020. 


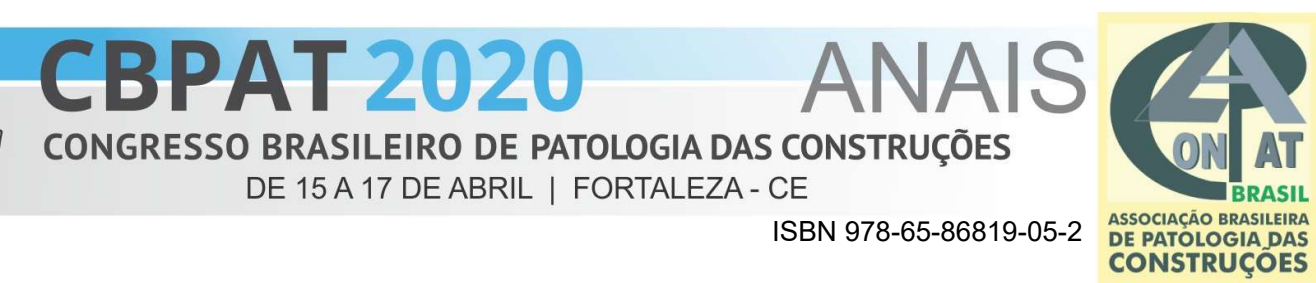

ZHIYONG, A.; SUN, W.; JIANG, J.; SONG, D.; MA, H.; ZHANG, J.; WANG, D. Passivation Characteristics of Alloy Corrosion-Resistant Steel Cr10Mo1 in Simulating Concrete Pore Solutions : Combination Effects of $\mathrm{pH}$ and Chloride. Materials, v. 9, 749, 2016. 